\title{
Aluminum triflate catalyzed Povarov reaction for the synthesis of pyranotetrahydroquinolines
}

\author{
Mokela S. Sokamisa, Yonela M. Nyondlo, and Henok H. Kinfe* \\ University of Johannesburg, Department of Chemistry, PO box 524, Auckland Park 2006, \\ South Africa \\ E-mail:hhkinfe@uj.ac.za
}

DOI: http://dx.doi.org/10.3998/ark.5550190.p009.447

\begin{abstract}
An efficient three component Povarov reaction is developed for the synthesis of pyrano[3,2-c]tetrahydroquinolines using a catalytic amount of $\mathrm{Al}(\mathrm{OTf})_{3}$ as a Lewis acid catalyst, in good to excellent yields.
\end{abstract}

Keywords: $\mathrm{Al}(\mathrm{OTf})_{3}$, Povarov reaction, pyranotetrahydroquinolines

\section{Introduction}

Pyranotetrahydroquinolines are important structural motifs that are present as substructures in a number of natural products and interesting biologically active compounds that are reported to possess psychotropic, antiallergic, anti-inflammatory, and estrogenic activities. ${ }^{1-5}$ Owing to their importance, a number of synthetic strategies have been developed for their synthesis. ${ }^{1,6-21}$ The reaction of an aromatic imine (in a two component reaction) or aniline (in a three component reaction) with benzaldehyde and electron rich dihydropyran (DHP) in the presence of an acid catalyst/promotor, known as the Povarov reaction, ${ }^{1,19-24}$ has been the common and convenient synthetic protocol for the preparation of such important compounds. Several acid catalysts/promoters, which include the following, have been reported in the literature: $\mathrm{InCl}_{3},{ }^{9}$ $\mathrm{Sc}(\mathrm{OTf})_{3},{ }^{25} \mathrm{I}_{2},{ }^{17}$ Ferric sulfate, ${ }^{18}$ montmorillonite $\mathrm{KSF},{ }^{11}$ lanthanide halides, ${ }^{13} \mathrm{BF}_{3} \cdot \mathrm{OEt}_{2},{ }^{22}$ $\mathrm{Sm}(\mathrm{OTf})_{3},{ }^{26} \mathrm{ZrCl}_{4},{ }^{23} \mathrm{CF}_{3} \mathrm{CO}_{2} \mathrm{H}^{8}{ }^{8} \mathrm{SnCl}_{2} \cdot 2 \mathrm{H}_{2} \mathrm{O},{ }^{28}$ polyaniline- $p$-toluenesulfonate salt, ${ }^{3}$ and cellulose sulfuric acid, ${ }^{15} \mathrm{VCl}_{3},{ }^{29} \mathrm{CAN}^{30} \mathrm{SmI}_{2}{ }^{31}$ Selectfluor ${ }^{\mathrm{TM}},{ }^{32}$ Tritylium Cation, ${ }^{33}$ and phosphoric acid. $^{34,35}$ However, for the Lewis acid catalysed three-component Povarov reaction, the need for a stoichiometric amount of the catalyst, due to the decomposition or deactivation of the acid by the amines (both in the reactant and product) and the production of water during the intermediate imine formation, has been reported as the main limitation of the protocol. ${ }^{3,10,11}$ 
Thus developing new Lewis acid catalysts that are stable under the reaction conditions and that can catalyse the reaction at a lower catalyst loading is desirable. Williams and Cullen reported that $\mathrm{Al}(\mathrm{OTf})_{3}$ was an effective recyclable catalyst $(<10 \mathrm{~mol} \%$ catalyst loading) for the ring opening of epoxides with amines to provide $\beta$-amino alcohols without the catalyst being deactivated by the nucleophile amine or hydroxyl amine product. ${ }^{36}$ Inspired by this result and in continuation of our research into the application of $\mathrm{Al}(\mathrm{OTf})_{3}$ in a number of catalytic transformations such as in the synthesis of chiral O-heterocycles, ${ }^{37}$ tetrahydropyranyl and tetrahydrofuranyl protection/deprotection of alcohols ${ }^{38}$ and temperature-switched selective Oglycosylation, ${ }^{39}$ we have evaluated its catalytic activity in the three component Povarov reaction and herein report its scope and limitations.

\section{Results and Discussion}

In our initial studies of the solvent-free $\mathrm{Al}(\mathrm{OTf})_{3}$ catalyzed three component Povarov reaction, we examined the reaction of aniline 1a (1 equivalent), benzaldehyde 2a (1 equivalent) and DHP ( 2 equivalents) in the presence of $5 \mathrm{~mol} \% \mathrm{Al}(\mathrm{OTf})_{3}$ at room temperature that gave the expected pyranotetrahydroquinolines $\mathbf{4 a}$ and $\mathbf{5 a}$ in 76\% combined yield, in $25 \mathrm{~min}$, as a 75:25 cis:trans ratio (Scheme 1). Encouraged by this result, the catalyst loading was reduced to $2 \mathrm{~mol} \%$ to make it more economical and environmentally friendly. The outcome however was that the reaction became sluggish.

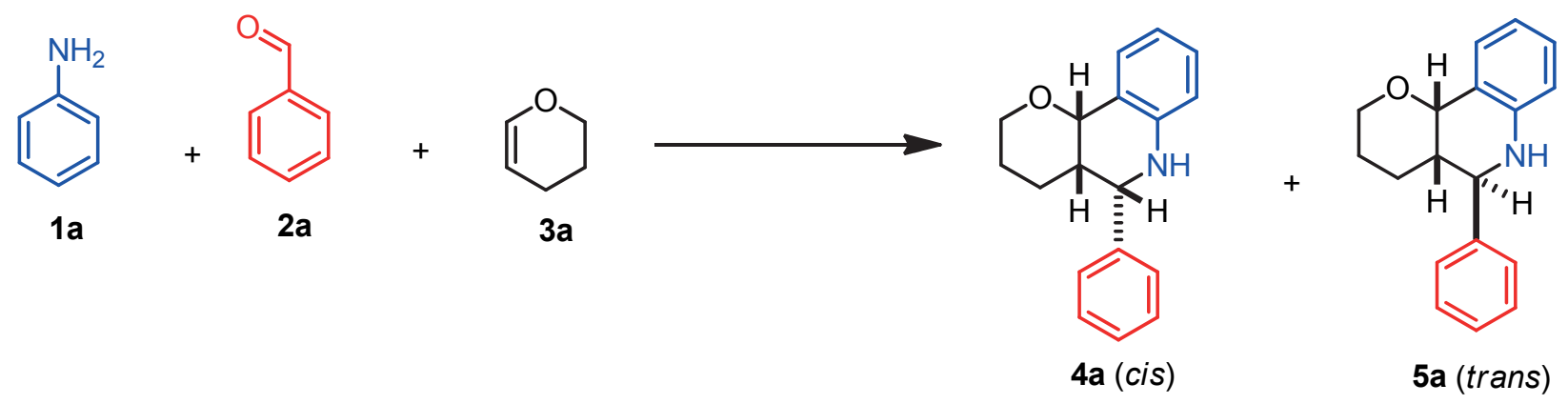

Scheme 1. $5 \mathrm{~mol} \% \mathrm{Al}(\mathrm{OTf})_{3}$, room temperature, $25 \mathrm{~min}, 76 \%$ (75:25 ratio).

Hoping to improve the diastereoisomeric ratio in favour of the kinetic product $4 \mathbf{a}^{3}{ }^{3}$ the reaction was attempted at $0{ }^{\circ} \mathrm{C}$ using $5 \mathrm{~mol} \% \mathrm{Al}(\mathrm{OTf})_{3}$. Unfortunately the reaction mixture became too viscous and even at higher temperatures $\left(40{ }^{\circ} \mathrm{C}, 60{ }^{\circ} \mathrm{C}\right.$ and $\left.80{ }^{\circ} \mathrm{C}\right)$ no improvement of selectivity in favour of the thermodynamic product ${ }^{3} \mathbf{5 a}$ took place. Next, we examined the role of the amount of DHP and it was found that 1.5 equivalents of the DHP (relative to aniline) were sufficient to provide the corresponding pyranotetrahydroquinolines $4 \mathbf{a}$ and $\mathbf{5 a}$ without significant effect on the yield and reaction time. The stereochemistries of the cis and trans isomers were established on the basis of the $J$ value of H-5 in each isomer. The smaller ${ }^{3} J_{\mathrm{H}-4 \mathrm{a}, \mathrm{H}-5}$ value of 5.6 
$\mathrm{Hz}$ indicates the cis relationship between $\mathrm{H}-4 \mathrm{a}$ and $\mathrm{H}-5$ while the larger ${ }^{3} \mathrm{~J}_{\mathrm{H}-4 \mathrm{a}, \mathrm{H}-5}$ value of $8.0 \mathrm{~Hz}$ suggests a trans relationship between these two protons. ${ }^{10}$

Having established the optimum reaction conditions, we then evaluated the scope of the reaction for various aniline and benzaldehyde derivatives. However, in most cases the reaction mixture solidified after the addition of the $\mathrm{Al}(\mathrm{OTf})_{3}$ without the formation of the expected pyranotetrahydroquinolines. This limitation led us to conduct re-optimization of the reaction in solvents which were previously reported to be suitable for the Povarov reaction. ${ }^{19-21}$ The solvents screened include acetonitrile, methylene chloride, THF, and toluene. Reactions were carried out by treating a solution of aniline, benzaldehyde and DHP in either THF or toluene with 5-20 $\mathrm{mol}_{\%} \mathrm{Al}(\mathrm{OTf})_{3}$ but at room temperature or higher $\left(60-80{ }^{\circ} \mathrm{C}\right)$ no formation of pyranotetrahydroquinolines $4 \mathbf{a}$ and $\mathbf{5 a}$ was observed. When reactions carried out in methylene chloride pyranotetrahydroquinolines $4 \mathbf{a}$ and $5 \mathbf{a}$ were obtained in $57 \%$ yield after $3.5 \mathrm{~h}$ with a 50:50 cis:trans selectivity at $50{ }^{\circ} \mathrm{C}$. No product formation was detected at room temperature. Interestingly, employing acetonitrile and carrying out the reaction at room temperature the pyranotetrahydroquinolines $\mathbf{4 a}$ and $\mathbf{5 a}$ were obtained in 62\% yield and 56:44 cis:trans ratio in 30 min. Since acetonitrile provided a better yield and shorter reaction time, it was identified as a suitable solvent for the $\mathrm{Al}(\mathrm{OTf})_{3}$ catalyzed Povarov reaction.

Having identified the best solvent and catalyst loading of the $\mathrm{Al}(\mathrm{OTf})_{3}$ which was $5 \mathrm{~mol} \%$ (lower catalyst loading resulted in slower reaction rates), we then examined the effect of temperature on the selectivity of the reaction. While reactions at $0{ }^{\circ} \mathrm{C}$ resulted in formation of $4 \mathbf{a}$ and $\mathbf{5 a}$ in poor yield (48\%) but better selectivity (70:30) in $3 \mathrm{~h}$, reactions at $50{ }^{\circ} \mathrm{C}$, the optimum temperature for the reaction, provided $70 \%$ of $\mathbf{4 a}$ and $\mathbf{5 a}$ in $48: 52$ ratio in $15 \mathrm{~min}$. Below $50{ }^{\circ} \mathrm{C}$ reactions were slower whereas there was no change in yield and reaction rate for reactions $>50$ ${ }^{\circ} \mathrm{C}$.

Next, we examined the scope of the modified reaction using various aniline and benzaldehyde derivatives. Electron-donating and electron-withdrawing groups on the aryl ring of the benzaldehyde and aniline were compatible under the reaction conditions and the corresponding pyranotetrahydroquinolines $\mathbf{4 a - i}$ and $\mathbf{5 a - i}$ were obtained in reasonable to excellent yields with selectivities ranging from 50:50 to 22:78 in favour of the trans diastereoisomer except for $\mathbf{4 b} / \mathbf{5 b}$ and $\mathbf{4 f} / \mathbf{5} \mathbf{f}$, which possess an electron donating group. The results are summarized in Table $\mathbf{1}$ with the products identified by ${ }^{1} \mathrm{H}$ and ${ }^{13} \mathrm{C}$ NMR. Moreover, the spectroscopic data were in agreement with the reported literature (Table 1).

For the lowest yielding reaction (Table 1, products $\mathbf{4 f}$ and $\mathbf{5 f}$ ), microwave irradiation efficiently enhanced the reaction time and provided a better yield at the expense of poorer selectivity. The yield of the pyranotetrahydroquinolines $4 \mathbf{f}$ and $\mathbf{5 f}$ improved from $43 \%$ after stirring for $45 \mathrm{~min}$ to $81 \%$ in under 6 min reaction time with the selectivity going down from 60:40 to 50:50 cis:trans ratio. The enhancement of the reaction rate and yield upon microwave irradiation was also observed with other substrates as indicated in Table 1. In most cases the yields of the reactions improved, although the selectivity of the reactions was found to be unpredictable; however, the reaction rate increased in all cases (Table 1). 
Table 1. The Povarov reaction of various aniline and aldehyde derivatives using $5 \mathrm{~mol} \%$ $\mathrm{Al}(\mathrm{OTf})_{3}$, in $\mathrm{CH}_{3} \mathrm{CN}$ at $50{ }^{\circ} \mathrm{C} / 70{ }^{\circ} \mathrm{C} \mathrm{MW}$ irradiation

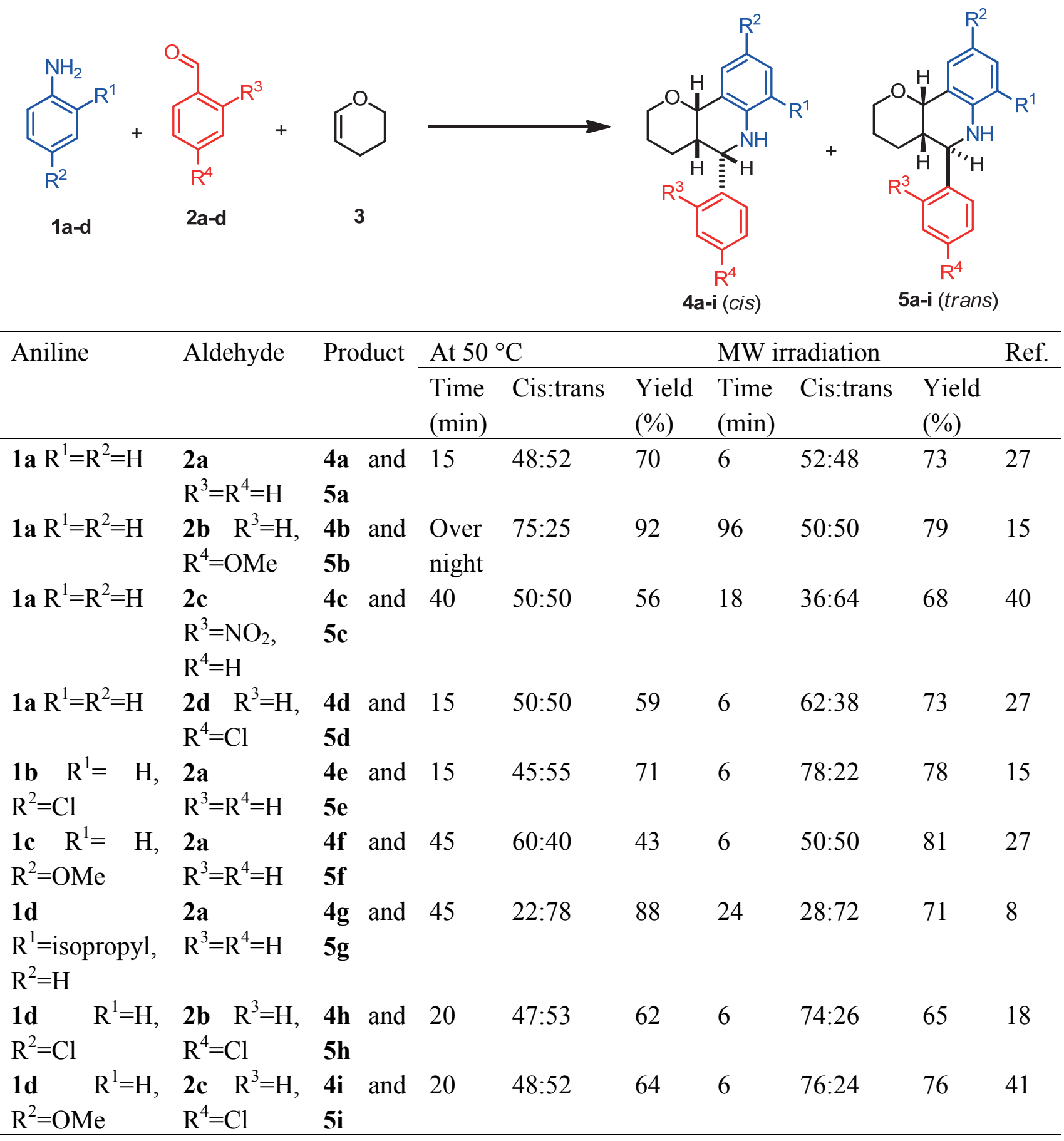


Comparison of $\mathrm{Al}(\mathrm{OTf})_{3}$ to other literature reported catalysts ${ }^{18}$ indicates that the current methodology is superior in terms of the reaction time and catalyst loading but comparable in selectivity and slightly inferior in yield, especially without microwave irradiation. (Table 2).

Table 2. Comparison of the current catalyst system to other reported systems ${ }^{18}$

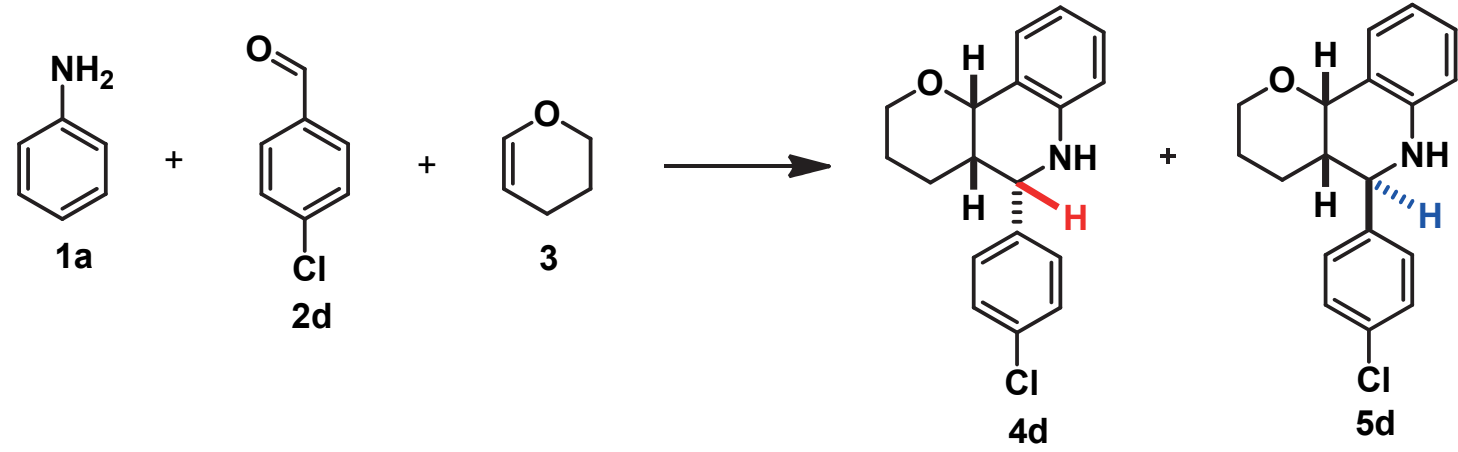

\begin{tabular}{|c|c|c|c|c|c|c|}
\hline Entry & Catalyst & Amount & Condition & Time & 4d:5d & Yield \% \\
\hline 1 & $\mathrm{FeCl}_{3}$ & $10 \mathrm{~mol} \%$ & RT & $6 \mathrm{~h}$ & $50: 50$ & $82^{\mathrm{a}}$ \\
\hline 2 & Proline triflate & $5 \mathrm{~mol} \%$ & RT & $5 \mathrm{~h}$ & $25: 75$ & $85^{\mathrm{a}}$ \\
\hline 3 & $\begin{array}{l}\text { 4-Nitrophthalic } \\
\text { acid }\end{array}$ & $25 \mathrm{~mol} \%$ & $50^{\circ} \mathrm{C}$ & $3.5 \mathrm{~h}$ & $39: 61$ & $90^{\mathrm{a}}$ \\
\hline 4 & $\mathrm{I}_{2}$ & $30 \mathrm{~mol} \%$ & RT & $3 \mathrm{~h}$ & $23: 77$ & $84^{\mathrm{a}}$ \\
\hline 5 & $\mathrm{Fe}_{2}\left(\mathrm{SO}_{4}\right)_{3} \cdot x \mathrm{H}_{2} \mathrm{O}$ & $10 \mathrm{~mol} \%$ & Reflux & $1.5 \mathrm{~h}$ & $19: 81$ & $87^{\mathrm{a}}$ \\
\hline 6 & $\mathbf{A l}(\mathbf{O T f})_{3}$ & $5 \mathrm{~mol} \%$ & $50{ }^{\circ} \mathbf{C}$ & $15 \mathrm{~min}$ & $50: 50$ & $59^{b}$ \\
\hline 7 & $\mathrm{Al}(\mathrm{OTf})_{3}$ & $5 \mathrm{~mol} \%$ & MW & $6 \mathrm{~min}$ & $62: 38$ & $73^{b}$ \\
\hline
\end{tabular}

${ }^{\mathrm{a}}$ Comparative data extracted from reference $18 .{ }^{\mathrm{b}}$ Current study.

\section{Conclusions}

In conclusion, a procedure for the synthesis of pyranotetrahydroquinolines has been demonstrated using a catalytic amount of $\mathrm{Al}(\mathrm{OTf})_{3}$ as a catalyst in the three-component Povarov reaction. The high yields obtained, short reaction times and the need for only a catalytic amount $(5 \mathrm{~mol} \%)$ of $\mathrm{Al}(\mathrm{OTf})_{3}$ in the reactions are clear indications that, unlike many other Lewis acids, $\mathrm{Al}(\mathrm{OTf})_{3}$ is not deactivated by the amine and water present in the reaction. Thus, the latter can be considered a suitable catalyst for the Povarov reaction for the preparation of pyranotetrahydroquinolines. 


\section{Experimental Section}

General. To a solution of aniline $1(1.074 \mathrm{mmol})$, benzaldehyde $2(1.074 \mathrm{mmol})$ and DHP 3 $(2.147 \mathrm{mmol})$ in acetonitrile $(4 \mathrm{~mL})$ was added $\mathrm{Al}(\mathrm{OTf})_{3}(5 \mathrm{~mol} \%, 0.054 \mathrm{mmol})$. The reaction mixture was stirred at $50{ }^{\circ} \mathrm{C}$ or under microwave irradiation at $70{ }^{\circ} \mathrm{C}$ until TLC analysis indicated completion of the reaction (refer to Table 1 for reaction times and yields). After completion of the reaction, the reaction mixture was allowed to cool down to room temperature and quenched by addition of saturated $\mathrm{NaHCO}_{3}$ solution $(5 \mathrm{~mL})$. The mixture was extracted with ethyl acetate $(3 \times 10 \mathrm{~mL})$. The combined organic layers were dried over $\mathrm{Na}_{2} \mathrm{SO}_{4}$, concentrated in vacuo and purified by column chromatography on silica gel using a mixture of ethyl acetate:hexane (1:9) as eluent to provide the corresponding pyranotetrahydroquinolines.

All microwave irradiation experiments were carried out in a multimode Multiwave PRO microwave reactor at $70{ }^{\circ} \mathrm{C}$ and $600 \mathrm{~W}$. This apparatus was used in the standard configuration as delivered. Reactions were carried out in sealed quartz glass vessels (using $8 \mathrm{~mL}$ of acetonitrile in an $80 \mathrm{~mL}$ vessel) and temperature control was done using external infrared sensor. The reaction mixture was continuously stirred until TLC analysis confirmed completion of the reaction (usually at $6 \mathrm{~min}$ interval). After the reaction is complete, the reaction vessel is cooled by forcedair cooling system to room temperature.

(4aR,5R,10bR)-5-Phenyl-3,4,4a,5,6,10b-hexahydro-2H-pyrano[3,2-c]quinolone (4a). White solid; mp 120-125 ${ }^{\circ} \mathrm{C}$ (Lit. $\left.{ }^{31} 123-124{ }^{\circ} \mathrm{C}\right) ; \delta_{\mathrm{H}}\left(400 \mathrm{MHz}, \mathrm{CDCl}_{3}\right)$ 7.49-7.30 (m, 6H, Ar), 7.10 (t, $J 7.4 \mathrm{~Hz}, 1 \mathrm{H}, \mathrm{Ar}), 6.82(\mathrm{t}, J 7.4 \mathrm{~Hz}, 1 \mathrm{H}, \mathrm{Ar}), 6.62(\mathrm{~d}, J 8.0 \mathrm{~Hz}, 1 \mathrm{H}, \mathrm{Ar}), 5.33(\mathrm{~d}, J 5.6 \mathrm{~Hz}, 1 \mathrm{H}, \mathrm{H}-$ 5), 4.68 (d, $J 2.0 \mathrm{~Hz}, 1 \mathrm{H}, \mathrm{H}-10 \mathrm{~b}$ ), 3.88 (bs, $1 \mathrm{H}, \mathrm{NH}$ ), 3.59 (dd, $J 1.6 \mathrm{~Hz}$ and $9.2 \mathrm{~Hz}, 1 \mathrm{H}, \mathrm{H}-2 \mathrm{a}$ ), 3.46 (td, $J 11.6 \mathrm{~Hz}$ and $2.4 \mathrm{~Hz}, 1 \mathrm{H}, \mathrm{H}-2 \mathrm{~b}$ ), 2.25-2.15 (m, $1 \mathrm{H}, \mathrm{H}-4 \mathrm{a}), 1.65-1.25$ (m, 4H, 2x CH${ }_{2}$ ), $\delta_{\mathrm{C}}\left(100 \mathrm{MHz}, \mathrm{CDCl}_{3}\right) 145.1,141.1,128.3,128.0,127.6,127.5,126.8,119.9,118.2,114.4,72.7$, $60.6,59.3,38.9,25.4,18.0$.

\section{(4aR,5S,10bR)-5-Phenyl-3,4,4a,5,6,10b-hexahydro-2H-pyrano[3,2-c]quinolone}

(5a). Colorless oil; $\delta_{\mathrm{H}}\left(400 \mathrm{MHz}, \mathrm{CDCl}_{3}\right) 7.50-7.31(\mathrm{~m}, 5 \mathrm{H}, \mathrm{Ar}), 7.25$ (d, $\left.J 6.4 \mathrm{~Hz}, 1 \mathrm{H}, \mathrm{Ar}\right), 7.11(\mathrm{t}, J$ $7.6 \mathrm{~Hz}, 1 \mathrm{H}, \mathrm{Ar}), 6.73(\mathrm{t}, J 7.4 \mathrm{~Hz}, 1 \mathrm{H}, \mathrm{Ar}), 6.42(\mathrm{~d}, J 8.0 \mathrm{~Hz}, 1 \mathrm{H}, \mathrm{Ar}), 4.73(\mathrm{~d}, J 8.0 \mathrm{~Hz}, 1 \mathrm{H}, \mathrm{H}-$ 5), $4.41(\mathrm{~d}, J 2.8 \mathrm{~Hz}, 1 \mathrm{H}, \mathrm{H}-10 \mathrm{~b}), 4.12(\mathrm{dt}, J 11.2 \mathrm{~Hz}$ and $2.0 \mathrm{~Hz}, 1 \mathrm{H}, \mathrm{H}-2 \mathrm{a}), 3.76(\mathrm{td}, J 11.6 \mathrm{~Hz}$ and $2.4 \mathrm{~Hz}, 1 \mathrm{H}, \mathrm{H}-2 \mathrm{~b}), 2.17-1.35\left(\mathrm{~m}, 4 \mathrm{H}, 2 \mathrm{x} \mathrm{CH}_{2}\right)$; $\delta_{\mathrm{C}}\left(100 \mathrm{MHz}, \mathrm{CDCl}_{3}\right) 144.8,142.4,130.9$, $129.4,128.7,127.9,127.8,120.7,117.5,114.1,68.6,54.9,38.9,24.2,22.1$. (4aR,5R,10bR)-5-(4-Methoxyphenyl)-3,4,4a,5,6,10b-hexahydro-2H-pyrano[3,2-c]quinoline (4b). White solid; mp $153-157{ }^{\circ} \mathrm{C}$ (Lit. $\left.{ }^{15} 120-123{ }^{\circ} \mathrm{C}\right) ; \delta_{\mathrm{H}}\left(400 \mathrm{MHz}, \mathrm{CDCl}_{3}\right) 7.41(\mathrm{~d}, J 7.6 \mathrm{~Hz}$, 1H, Ar), 7.32 (d, J 8.4 Hz, 2H, Ar), 7.08 (t, J 7.6 Hz, 1H, Ar), 6.90 (d, J 8.4 Hz, 2H, Ar), 6.77 (t, $J 7.4 \mathrm{~Hz}, 1 \mathrm{H}, \mathrm{Ar}), 6.58(\mathrm{~d}, J 8.0 \mathrm{~Hz}, 1 \mathrm{H}, \mathrm{Ar}), 5.30$ (d, $J 5.6 \mathrm{~Hz}, 1 \mathrm{H}, \mathrm{H}-5), 4.63$ (d, $J 2.0 \mathrm{~Hz}, 1 \mathrm{H}$, $\mathrm{H}-10 \mathrm{~b}), 3.81$ (bs, $4 \mathrm{H}, \mathrm{NH}$ and $\left.\mathrm{OCH}_{3}\right), 3.61-3.54(\mathrm{~m}, 1 \mathrm{H}, \mathrm{H}-2 \mathrm{a}), 3.46-3.35$ (m, 1H, H-2b), 2.14$2.05(\mathrm{~m}, 1 \mathrm{H}, \mathrm{H}-4 \mathrm{a}), 1.55-1,28\left(\mathrm{~m}, 4 \mathrm{H}, 2 \mathrm{x} \mathrm{CH}_{2}\right) ; \delta_{\mathrm{C}}\left(100 \mathrm{MHz}, \mathrm{CDCl}_{3}\right)$ 158.9, 145.2, 133.1, $128.0,127.9,127.6,119.9,118.2,114.4,113.6,72.7,60.6,58.7,55.3,39.0,25.4,18.0$. 
(4a $R, 5 S, 10 \mathrm{~b} R)$-5-(4-Methoxyphenyl)-3,4,4a,5,6,10b-hexahydro-2H-pyrano[3,2-c]quinoline

(5b). White solid; mp $115-117^{\circ} \mathrm{C}$ (Lit. $\left.{ }^{15} 100-103{ }^{\circ} \mathrm{C}\right) ; \delta_{\mathrm{H}}\left(400 \mathrm{MHz}, \mathrm{CDCl}_{3}\right) 7.32(\mathrm{~d}, J 8.4 \mathrm{~Hz}$, 2H, Ar), 7.21 (d, J 7.6 Hz, 1H, Ar), 7.10-7.00 (m, 1H, Ar), 6.90 (d, J 8.8 Hz, 2H, Ar), 6.68 (t, $J$ $7.2 \mathrm{~Hz}, 1 \mathrm{H}, \mathrm{Ar}), 6.50$ (d, J 8.0 Hz, 1H, Ar), 4.66 (d, J $10.8 \mathrm{~Hz}, 1 \mathrm{H}, \mathrm{H}-5), 4.38$ (d, J $2.4 \mathrm{~Hz}, 1 \mathrm{H}$, $\mathrm{H}-10 \mathrm{~b}), 4.15-4.05$ (m, 1H, H-2a), 3.81 (s, 3H, OCH $), 3.71(\mathrm{td}, J 3.6 \mathrm{~Hz}$ and $2.0 \mathrm{~Hz}, 1 \mathrm{H}, \mathrm{H}-2 \mathrm{~b})$, 2.10-2.00 (m, 1H, H-4a), 1.90-1.60 (m, 2H, $\left.\mathrm{CH}_{2}\right), 1.53-1.25\left(\mathrm{~m}, 2 \mathrm{H}, \mathrm{CH}_{2}\right) ; \delta_{\mathrm{C}}(100 \mathrm{MHz}$, $\left.\mathrm{CDCl}_{3}\right)$ 159.2, 130.9, 129.3, 129.0, 128.2, 126.0, 125.7, 125.2, 113.94, 68.7, 55.2, 54.1, 38.8, 24.1, 21.9.

(4aR,5R,10bR)-5-(2-Nitrophenyl)-3,4,4a,5,6,10b-hexahydro-2H-pyrano[3,2-c]quinoline (4c). Yellow solid; mp 202-206 ${ }^{\circ} \mathrm{C}\left(\right.$ Lit. $\left.^{29} 203-204{ }^{\circ} \mathrm{C}\right) ; \delta_{\mathrm{H}}\left(400 \mathrm{MHz}, \mathrm{CDCl}_{3}\right) 7.95(\mathrm{t}, J 8.4 \mathrm{~Hz}, 2 \mathrm{H}$, Ar), 7.63 (t, J 7.4 Hz, 1H, Ar), 7.49-7.40 (m, 2H, Ar), 7.08 (t, J 7.4 Hz, 1H, Ar), 6.82 (t, J 7.4 Hz, 1H, Ar), 6.59 (d, J 8.0 Hz, 1H, Ar), 5.32 (d, J 5.6 Hz, 1H, H-5), 5.14 (s, 1H, H-10b), 3.68 (s, 1H, NH), 3.55 (bd, J 10.4 Hz, 1H, H-2a), 3.41 (t, J 11.4 Hz, 1H, H-2b), 2.55-2.45 (m, 1H, H-4a), 1.69-1.26 (m, 4H, 2x CH$) ; \delta_{\mathrm{C}}\left(100 \mathrm{MHz} \mathrm{CDCl}_{3}\right) 148.8,144.8,135.7,132.6,129.4,128.3$, $128.1,127.8,120.4,119.0,114.9,72.3,60.5,54.5,36.2,25.3,18.5$.

(4aR,5S,10bR)-5-(2-Nitrophenyl)-3,4,4a,5,6,10b-hexahydro-2 $H$-pyrano[3,2-c]quinoline (5c). Yellow solid; mp 211-214 ${ }^{\circ} \mathrm{C}$; $\delta_{\mathrm{H}}\left(400 \mathrm{MHz}, \mathrm{CDCl}_{3}\right) 7.80(\mathrm{~d}, J 8.0 \mathrm{~Hz}, 1 \mathrm{H}, \mathrm{Ar}), 7.65(\mathrm{~d}, J 7.6$ $\mathrm{Hz}, 1 \mathrm{H}, \mathrm{Ar}), 7.60-7.50$ (m, 1H, Ar), 7.45-7.35 (m, 1H, Ar), 7.25 (d, J 8.0 Hz, 1H, Ar), 7.15-7.05 $(\mathrm{m}, 1 \mathrm{H}, \mathrm{Ar}), 6.74(\mathrm{td}, J 7,6 \mathrm{~Hz}$ and $0.8 \mathrm{~Hz}, 1 \mathrm{H}, \mathrm{Ar}), 6.57(\mathrm{~d}, J 8.0 \mathrm{~Hz}, 1 \mathrm{H}, \mathrm{Ar}), 5.12(\mathrm{~d}, J 8.0 \mathrm{~Hz}$, 1H, H-5), 4.45 (d, J 3.6 Hz, 1H, H-10b), 4.24 (bs, 1H, NH), 3.90-3.81 (m, 1H, H-2a), 3.67-3.57 (m, 1H, H-2b), 2.25-2.15 (m, 1H, H-4a), 1.81-1.38 (m, 4H, 2 x CH$) ; \delta_{\mathrm{C}}\left(100 \mathrm{MHz}, \mathrm{CDCl}_{3}\right)$ $149.9,143.9,132.8,129.8,129.3,129.1,128.4,124.0,119.8,118.0,114.0,72.2,66.0,50.8,37.7$, 24.5, 23.1 .

$(4 \mathrm{a} R, 5 R, 10 \mathrm{~b} R)$-5-(4-chlorophenyl)-3,4,4a,5,6,10b-hexahydro-2H-pyrano[3,2-c]quinoline (4d). White solid mp $170-173{ }^{\circ} \mathrm{C}\left(\right.$ Lit. $\left.^{18} 170{ }^{\circ} \mathrm{C}\right) ; \delta_{\mathrm{H}}\left(400 \mathrm{MHz}, \mathrm{CDCl}_{3}\right) 7.41(\mathrm{~d}, J 7.6 \mathrm{~Hz}, 1 \mathrm{H}$, Ar), 7.34 (s, 4H, Ar), 7.13-7.00 (m, 1H, Ar), 6.80 (t, J $7.6 \mathrm{~Hz}, 1 \mathrm{H}, \mathrm{Ar}), 6.54$ (d, J 8.0 Hz, 1H, Ar), 5.30 (d, J $5.2 \mathrm{~Hz}, 1 \mathrm{H}, \mathrm{H}-5), 4.65$ (d, J $2.4 \mathrm{~Hz}, 1 \mathrm{H}, \mathrm{H}-10 \mathrm{~b}), 3.82$ (bs, 1H, NH), 3.57 (dd, $J$ $10.0 \mathrm{~Hz}$ and $8.0 \mathrm{~Hz}, 1 \mathrm{H}, \mathrm{H}-2 \mathrm{a}), 3.45-3.35$ (m, 1H, H-2b), 2.15-2.05 (m, 1H, H-4a), 1.60-1.20 $\left(\mathrm{m}, 4 \mathrm{H}, 2 \times \mathrm{CH}_{2}\right) ; \delta_{\mathrm{C}}\left(100 \mathrm{MHz}, \mathrm{CDCl}_{3}\right) 144.9,139.6,133.1,128.5,128.1,127.6,119.1,118.6$, $114.5,72.6,60.6,58.8,38.9,25.3,17.9$.

(4a $R, 5 S, 10 \mathrm{~b} R$ )-5-(4-Chlorophenyl)-3,4,4a,5,6,10b-hexahydro-2H-pyrano[3,2-c]quinoline

(5d). White solid; mp $125-130{ }^{\circ} \mathrm{C}$ (Lit. $\left.{ }^{18} 124{ }^{\circ} \mathrm{C}\right) ; \delta_{\mathrm{H}}\left(400 \mathrm{MHz}, \mathrm{CDCl}_{3}\right) 7.34$ (bs, 4H, Ar), 7.21 (d, J 7.6 Hz, 1H, Ar), 7.09 (t, J 7.6 Hz, 1H, Ar), 6.71 (t, J 7.4 Hz, 1H, Ar), 6.52 (d, J 8.0 Hz, 1H, Ar), $4.68(\mathrm{~d}, J 10.8 \mathrm{~Hz}, 1 \mathrm{H}, \mathrm{H}-5), 4.37$ (d, $J 2.4 \mathrm{~Hz}, 1 \mathrm{H}, \mathrm{H}-10 \mathrm{~b}), 4.12-3.95(\mathrm{~m}, 2 \mathrm{H}, \mathrm{NH}$ and H2a), $3.71(\mathrm{td}, J 11.6 \mathrm{~Hz}$ and $2.4 \mathrm{~Hz}, 1 \mathrm{H}, \mathrm{H}-2 \mathrm{~b}), 2.10-2.00(\mathrm{~m}, 1 \mathrm{H}, \mathrm{H}-4 \mathrm{a}), 1.87-1.55\left(\mathrm{~m}, 2 \mathrm{H}, \mathrm{CH}_{2}\right)$, $1.50-138\left(\mathrm{~m}, 2 \mathrm{H}, \mathrm{CH}_{2}\right) ; \delta_{\mathrm{C}}\left(100 \mathrm{MHz}, \mathrm{CDCl}_{3}\right) 144.5,140.9,133.5,130.9,129.4,129.1,128.8$, $120.7,117.7,114.2,74.3,68.5,54.3,38.9,24.0,22.0$.

(4a $R, 5 R, 10 b R)-9-C h l o r o-5-p h e n y l-3,4,4 a, 5,6,10 b-h e x a h y d r o-2 H$-pyrano[3,2-c]quinoline (4e). White solid; mp $168-172{ }^{\circ} \mathrm{C}$ (Lit. $\left.{ }^{15} 170-175^{\circ} \mathrm{C}\right) ; \delta_{\mathrm{H}}\left(400 \mathrm{MHz}, \mathrm{CDCl}_{3}\right)$ 7.40-7.35 (m, 6H, Ar), 7.05-6.95 (m, 1H, Ar), 6.51 (d, J 8.4 Hz, 1H, Ar), 5.25 (d, J 5.6 Hz, 1H, H-5), 4.64 (d, J 2.4 
$\mathrm{Hz}, 1 \mathrm{H}, \mathrm{H}-10 \mathrm{~b}), 3.88$ (s, 1H, NH), 3.65-3.55 (m, 1H, H-2a), 3.45-3.36 (m, 1H, H-2b), 2.20-2.10 $(\mathrm{m}, 1 \mathrm{H}, \mathrm{H}-4 \mathrm{a}), 1.55-1.35\left(\mathrm{~m}, 4 \mathrm{H}, 2 \times \mathrm{CH}_{2}\right) ; \delta_{\mathrm{C}}\left(100 \mathrm{MHz}, \mathrm{CDCl}_{3}\right)$ 143.7, 140.6, 128.4, 128.0, $127.7,127.6,126.7,123.0,121.6,115.6,72.4,60.8,59.3,38.5,25.3,18.0$.

(4a $R, 5 S, 10 b R)-9-C h l o r o-5-p h e n y l-3,4,4 a, 5,6,10 b-h e x a h y d r o-2 H$-pyrano[3,2-c]quinoline

(5e). Colorless oil; $\delta_{\mathrm{H}}\left(400 \mathrm{MHz}, \mathrm{CDCl}_{3}\right)$ 7.45-7.30 (m, 5H, Ar), 7.20 (d, J 2.4 Hz, 1H, Ar), 7.02 (dd, $J 8.4 \mathrm{~Hz}$ and $2.4 \mathrm{~Hz}, 1 \mathrm{H}, \mathrm{Ar}), 6.44$ (d, J 8.4 Hz, 1H, Ar), 4.66 (d, J $10.4 \mathrm{~Hz}, 1 \mathrm{H}, \mathrm{H}-5), 4.33$ (d, J $2.4 \mathrm{~Hz}, 1 \mathrm{H}, \mathrm{H}-10 \mathrm{~b}), 4.15-4.03(\mathrm{~m}, 2 \mathrm{H}, \mathrm{H}-2 \mathrm{a}$ and $\mathrm{NH}), 3.70(\mathrm{td}, J 11.2 \mathrm{~Hz}$ and $2.4 \mathrm{~Hz}, 1 \mathrm{H}$, $\mathrm{H}-2 \mathrm{~b}), 2.10-2.00(\mathrm{~m}, 1 \mathrm{H}, \mathrm{H}-4 \mathrm{a}), 1.90-1.40$ (m, 4H, 2 х CH$)$; $\delta_{\mathrm{C}}\left(100 \mathrm{MHz}, \mathrm{CDCl}_{3}\right)$ 143.3, 141.9, $130.3,129.2$, 127.98, 127.6, 121.8, 121.7, 115.3, 73.9, 68.4, 54.9, 38.6, 23.9, 22.0.

(4aR,5R,10bR)-9-Methoxy-5-phenyl-3,4,4a,5,6,10b-hexahydro-2H-pyrano[3,2-c]quinoline (4f). White solid; mp 144-147 ${ }^{\circ} \mathrm{C}$ (Lit. $\left.{ }^{18} 144^{\circ} \mathrm{C}\right) ; \delta_{\mathrm{H}}\left(400 \mathrm{MHz}, \mathrm{CDCl}_{3}\right)$ 7.40-7.25 (m, 5H, Ar), $7.02(\mathrm{~d}, J 2.8 \mathrm{~Hz}, 1 \mathrm{H}, \mathrm{Ar}), 6.71(\mathrm{dd}, J 8.4 \mathrm{~Hz}$ and $2.8 \mathrm{~Hz}, 1 \mathrm{H}, \mathrm{Ar}), 6.56(\mathrm{~d}, J 8.8 \mathrm{~Hz}, 1 \mathrm{H}, \mathrm{Ar})$, 5.30 (d, J $5.2 \mathrm{~Hz}, 1 \mathrm{H}, \mathrm{H}-5), 4.61$ (d, J $2.0 \mathrm{~Hz}, 1 \mathrm{H}, \mathrm{H}-10 \mathrm{~b}), 3.77$ (s, 3H, OCH $), 3.70-3.55$ (m, 2H, $\mathrm{NH}$ and H-2a), 3.42 (dt, $J 11.6 \mathrm{~Hz}$ and $2.4 \mathrm{~Hz}, 1 \mathrm{H}, \mathrm{H}-2 \mathrm{~b}), 2.22-2.08$ (m, 1H, H-4a), 1.60-1.20 $\left(\mathrm{m}, 4 \mathrm{H}, 2 \mathrm{X} \mathrm{CH}_{2}\right) ; \delta_{\mathrm{C}}\left(100 \mathrm{MHz}, \mathrm{CDCl}_{3}\right) 152.8,141.3,139.1,128.3,127.4,126.8,121.1,115.7$, $115.0,111.8,72.9,60.8,59.6,55.8,39.0,25.3,17.9$.

(4aR,5S,10bR)-9-Methoxy-5-phenyl-3,4,4a,5,6,10b-hexahydro-2H-pyrano[3,2-c]quinoline

(5f). Colorless oil; $\delta_{\mathrm{H}}\left(400 \mathrm{MHz}, \mathrm{CDCl}_{3}\right)$ 7.45-7.26 (m, 5H, Ar), 6.81 (d, J 2.8 Hz, 1H, Ar), 6.74 (dd, $J 8.4 \mathrm{~Hz}$ and $2.8 \mathrm{~Hz}, 1 \mathrm{H}, \mathrm{Ar}), 6.49$ (d, $J 8.4 \mathrm{~Hz}, 1 \mathrm{H}, \mathrm{Ar}), 4.65$ (d, $J 10.4 \mathrm{~Hz}, 1 \mathrm{H}, \mathrm{H}-5), 4.36$ (d, J 2.4 Hz, 1H, H-10b), 4.13-4.04 (m, 1H, H-2a), 3.88 (bs, 1H, NH), 3.80-3.65 (m, 4H, OCH and $\mathrm{H}-2 \mathrm{~b}), 2.13-2.04(\mathrm{~m}, 1 \mathrm{H}, \mathrm{H}-4 \mathrm{a}), 1.90-1.27$ (m, 4H, $\left.2 \times \mathrm{CH}_{2}\right) ; \delta_{\mathrm{C}}\left(100 \mathrm{MHz}, \mathrm{CDCl}_{3}\right)$ 152.0, $142.4,139.0,128.5,127.8,121.3,116.8,115.5,114.9,74.5,68.5,55.9,55.2,39.0,24.1,22.1$.

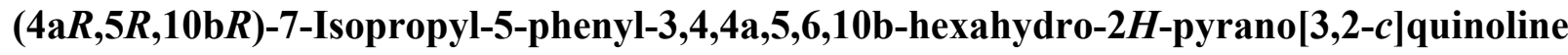
(4g). Colorless oil; $\delta_{\mathrm{H}}\left(400 \mathrm{MHz}, \mathrm{CDCl}_{3}\right)$ 7.49-7.30 (m, 6H, Ar), 7.08 (d, J 7.2 Hz, 1H, Ar), 6.80 (t, J 7.6 Hz, 1H, Ar), 5.36 (d, J $5.6 \mathrm{~Hz}, 1 \mathrm{H}, \mathrm{H}-5), 4.69$ (d, J $2.0 \mathrm{~Hz}, 1 \mathrm{H}, \mathrm{H}-10 \mathrm{~b}), 3.86$ (bs, 1H, $\mathrm{NH}), 3.60-3.51$ (m, 1H, H-2a), 3.50-3.38 (m, 1H, H-2b), 2.87-2.75 (m, 1H, $\left.\left(\mathrm{CH}_{3}\right)_{2} \mathrm{CH}\right), 2.20-$ $2.10(\mathrm{~m}, 1 \mathrm{H}, \mathrm{H}-4 \mathrm{a}), 1.60-1.35\left(\mathrm{~m}, 4 \mathrm{H}, 2 \times \mathrm{CH}_{2}\right), 1.30\left(\mathrm{~d}, J 6.8 \mathrm{~Hz}, 3 \mathrm{H}, \mathrm{CH}_{3}\right), 1.25(\mathrm{~d}, J 6.8 \mathrm{~Hz}$, $\left.3 \mathrm{H}, \mathrm{CH}_{3}\right) ; \delta_{\mathrm{C}}\left(100 \mathrm{MHz}, \mathrm{CDCl}_{3}\right) 141.7,141.5,131.8,128.4,127.5,126.8,125.1,124.0,119.8$, $118.0,60.6,59.2,38.6,27.5,25.4,22.4,22.1,18.0$.

$(4 \mathrm{a} R, 5 S, 10 \mathrm{~b} R)$-7-Isopropyl-5-phenyl-3,4,4a,5,6,10b-hexahydro-2H-pyrano[3,2-c]quinoline (5g). Colorless oil; $\delta_{\mathrm{H}}\left(400 \mathrm{MHz}, \mathrm{CDCl}_{3}\right) 7.50-7.32(\mathrm{~m}, 5 \mathrm{H}, \mathrm{Ar}), 7.13$ (t, J 6.8 Hz, 2H, Ar), 6.76 (t, J 7.6 Hz, 1H, Ar), 4.79 (d, J 11.2 Hz, 1H, H-5), 4.43 (d, J 2.4 Hz, 1H, H-10b), 4.20-4.05 (m, $2 \mathrm{H}, \mathrm{NH}$ and $\mathrm{H}-2 \mathrm{a}), 3.75$ (td, $J 12.0 \mathrm{~Hz}$ and $2.4 \mathrm{~Hz}, \mathrm{H}-2 \mathrm{~b}), 2.82-2.69\left(\mathrm{~m}, 1 \mathrm{H},\left(\mathrm{CH}_{3}\right)_{2} \mathrm{CH}\right), 2.15-$ 2.05 (m, 1H, H-4a), 1.97-1.82 (m, 1H, H-3a), 1.73-1.60 (m, 1H, H-3b), 1,53-1.30 (m, 2H, CH $)$, $1.21\left(\mathrm{~d}, J 6.8 \mathrm{~Hz}, 3 \mathrm{H}, \mathrm{CH}_{3}\right), 1.18\left(\mathrm{~d}, J 6.8 \mathrm{~Hz}, 3 \mathrm{H}, \mathrm{CH}_{3}\right) ; \delta_{\mathrm{C}}\left(100 \mathrm{MHz}, \mathrm{CDCl}_{3}\right)$ 142.8, 141.1, $131.2,128.6,127.8,127.8,125.2,120.3,117.1,68.7,54.8,38.7,27.0,24.0,22.3,22.0,21.9$.

(4aR,5R,10bR)-9-Chloro-5-(4-chlorophenyl)-3,4,4a,5,6,10b-hexahydro-2H-pyrano[3,2-

c]quinoline (4h). White solid; mp $188-192{ }^{\circ} \mathrm{C}\left(\mathrm{Lit}^{18} 188^{\circ} \mathrm{C}\right) ; \delta_{\mathrm{H}}\left(400 \mathrm{MHz}, \mathrm{CDCl}_{3}\right) 7.41(\mathrm{~d}, J$ $7.6 \mathrm{~Hz}, 1 \mathrm{H}, \mathrm{Ar}), 7.34$ (bs, 3H, Ar), 7.15-7.05 (m, 1H, Ar), 6.80-6.70 (m, 1H, Ar), 6.59 (d, J 7.6 Hz, 1H, Ar), 5.30 (d, J 5.6 Hz, 1H, H-5), 4.65 (d, J 2.4 Hz, 1H, H-10b), 3.81 (bs, 1H, NH), 3.63- 
$3.53(\mathrm{~m}, 1 \mathrm{H}, \mathrm{H}-2 \mathrm{a}), 3.41$ (td, $J 11.6 \mathrm{~Hz}$ and $2.4 \mathrm{~Hz}, 1 \mathrm{H}, \mathrm{H}-2 \mathrm{~b}), 2.16-2.06$ (m, 1H, H-4a), 1.58$1.02\left(\mathrm{~m}, 4 \mathrm{H}, 2 \times \mathrm{CH}_{2}\right) ; \delta_{\mathrm{C}}\left(100 \mathrm{MHz}, \mathrm{CDCl}_{3}\right) 143.1,140.5,130.4,129.3,129.0,128.9$, 122.1, $121.9,115.4,68.5,54.4,38.7,23.9,22.0$.

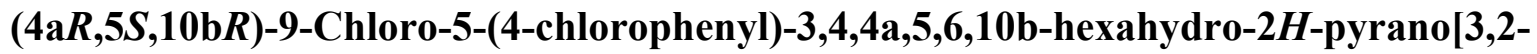
c]quinoline (5h). White solid; mp $154-156{ }^{\circ} \mathrm{C}\left(\mathrm{Lit}^{18}{ }^{152}{ }^{\circ} \mathrm{C}\right) ; \delta_{\mathrm{H}}\left(400 \mathrm{MHz}, \mathrm{CDCl}_{3}\right) 7.34(\mathrm{~s}, 4 \mathrm{H}$, Ar), $7.21(\mathrm{dd}, J 7.6 \mathrm{~Hz}$ and $1.2 \mathrm{~Hz}, 1 \mathrm{H}, \mathrm{Ar}), 7.08(\mathrm{td}, J 8.0 \mathrm{~Hz}$ and $1.6 \mathrm{~Hz}, 1 \mathrm{H}, \mathrm{Ar}), 6.71(\mathrm{td}, J$ $7.6 \mathrm{~Hz}$ and $0.8 \mathrm{~Hz}, 1 \mathrm{H}, \mathrm{Ar}), 6.52(\mathrm{~d}, J 8.0 \mathrm{~Hz}, 1 \mathrm{H}, \mathrm{Ar}), 4.68$ (d, J 10.8 Hz, 1H, H-5), 4.37 (d, J $2.8 \mathrm{~Hz}, 1 \mathrm{H}, \mathrm{H}-10 \mathrm{~b}), 4.12-3.97(\mathrm{~m}, 2 \mathrm{H}, \mathrm{NH}$ and $\mathrm{H}-2 \mathrm{a}), 3.71$ (td, $J 11.2 \mathrm{~Hz}$ and $2.4 \mathrm{~Hz}, \mathrm{H}-2 \mathrm{~b})$, 2.07-2.0 (m, H, H-4a), 1.90-1.60 (m, $\left.2 \mathrm{H}, \mathrm{CH}_{2}\right), 1.50-1.30\left(\mathrm{~m}, 2 \mathrm{H}, \mathrm{CH}_{2}\right) ; \delta_{\mathrm{C}}\left(100 \mathrm{MHz}, \mathrm{CDCl}_{3}\right)$ $144.9,139.6,133.1,128.5,128.4,128.1,127.6,119.9,118.6,114.5,72.6,60.6,58.8,38.9,25.3$, 17.9 .

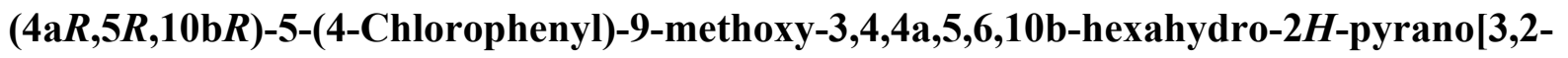
c)quinoline (4i). White solid; mp $158-164{ }^{\circ} \mathrm{C}$; $\delta_{\mathrm{H}}\left(400 \mathrm{MHz}, \mathrm{CDCl}_{3}\right)$ 7.40-7.30 (m, 4H, Ar), 7.01 (d, $J 2.4 \mathrm{~Hz}, 1 \mathrm{H}, \mathrm{Ar}), 6.71(\mathrm{dd}, J 8.4 \mathrm{~Hz}$ and $2.4 \mathrm{~Hz}, \mathrm{Ar}), 6.56$ (d, $J 8.8 \mathrm{~Hz}, 1 \mathrm{H}, \mathrm{Ar}), 5.27$ (d, $J$ $5.6 \mathrm{~Hz}, 1 \mathrm{H}, \mathrm{H}-5), 4.58$ (d, J 1.6 Hz, 1H, H-10b), 3.76 (s, 3H, OCH $), 3.65-3.55$ (m, $2 \mathrm{H}, \mathrm{NH}$ and H-2a), 3.50-3.30 (m, 1H, H-2b), 2.15-2.05 (m, 1H, H-4a), 1.60-1.15 (m, 4H, 2 x CH $)$; $\delta_{\mathrm{C}}(100$ $\left.\mathrm{MHz}, \mathrm{CDCl}_{3}\right) 153.6,140.0,138.9,133.1,128.5,128.3,121.3,116.0,115.2,111.9,72.9$, 60.9, 39.2, 25.4, 17.8 .

(4aR,5S,10bR)-5-(4-Chlorophenyl)-9-methoxy-3,4,4a,5,6,10b-hexahydro-2H-pyrano[3,2c)quinoline (5i). Light green oil; $\delta_{\mathrm{H}}\left(400 \mathrm{MHz}, \mathrm{CDCl}_{3}\right)$ 7.38-7.30 (m, 4H, Ar), 6.79 (d, J 2.4 Hz, $1 \mathrm{H}, \mathrm{Ar}), 6.73(\mathrm{dd}, J 8.8 \mathrm{~Hz}$ and $2.8 \mathrm{~Hz}, 1 \mathrm{H}, \mathrm{Ar}), 6.49$ (d, $J 8.4 \mathrm{~Hz}, 1 \mathrm{H}, \mathrm{Ar}), 4.62$ (d, J 10.8 Hz, 1H, H-5), 4.34 (d, J 2.4 Hz, 1H, H-10b), 4.07 (bd, J 11.2 Hz, 1H, H-2a), 3.83 (s, 1H, NH), 3.753.67 (m, 4H, O-CH $H_{3}$ and $\left.\mathrm{H}-2 \mathrm{~b}\right), 2.09-1.97$ (m, $\left.1 \mathrm{H}, \mathrm{H}-4 \mathrm{a}\right), 1.86-1.65$ (m, 2H, $\left.\mathrm{CH}_{2}\right), 1.48-1.27$ (m, $\left.2 \mathrm{H}, \mathrm{CH}_{2}\right) ; \delta_{\mathrm{C}}\left(100 \mathrm{MHz}, \mathrm{CDCl}_{3}\right) 152.2,141.0,138.5,129.2,128.8,121.4,117.0,115.7,114.8$, $68.6,56.0,39.1 ; 24.2 ; 22.1$.

\section{Acknowledgements}

We thank the University of Johannesburg (UJ), the Research Centre for Synthesis and Catalysis of the Department of Chemistry-UJ, the National Research Foundation (NRF) and Sasol Ltd for funding. The use of UJ Spectrum's NMR facilities is also acknowledged.

\section{References}

1. Sridharan, V.; Suryavanshi, P. A.; Menéndez, J. C. Chem. Rev. 2011, 111, 7157. http://dx.doi.org/10.1021/cr100307m

2. Camps, P.; Formosa, X.; Galdeano, C.; Muñoz-Torrero, D.; Ramírez, L.; Gómez, E.; Isambert, N.; Lavilla, R.; Badia, A.; Clos, M. V.; Bartolini, M.; Mancini, F.; Andrisano, V.; Arce, M. P.; Rodríguez-Franco, M. I.; Huertas, Ó.; Dafni, T.; Luque, F. J. J. Med. Chem. 2009, 52, 5365 . 
http://dx.doi.org/10.1021/jm900859q

3. Palaniappan, S.; Rajender, B.; Umashankar, M. J. Mol. Catal. A: Chem. 2012, 352, 70 and references cited therein. http://dx.doi.org/10.1016/j.molcata.2011.10.014

4. Majumdar, K. C.; Ponra, S.; Ghosh, T.; Sadhukhan, R.; Ghosh, U. Eur. J. Med. Chem. 2014, $71,306$. http://dx.doi.org/10.1016/j.ejmech.2013.10.067

5. Ghorab, M. M.; Abdel-Hamide, S. G.; Farrag, H. A. ACTA Pol. Pharm. 2001, 58, 175.

6. Zhang, Q.; Zhang, Z.; Yan, Z.; Liu, Q.; Wang, T. Org. Lett. 2007, 9, 3651. http://dx.doi.org/10.1021/o1062918m

7. Majumdar, K. C.; Ponra, S.; Ghosh, T. Tetrahedron Lett. 2013, 54, 5586. http://dx.doi.org/10.1016/j.tetlet.2012.11.017

8. Baudelle, R.; Melnyk, P.; Déprez, B.; Tartar, A. Tetrahedron 1998, 54, 4125. http://dx.doi.org/10.1016/S0040-4020(98)00140-9

9. Zhang, J.; Li, C. -J. J. Org. Chem. 2002, 67, 3969. http://dx.doi.org/10.1021/jo0161451

10. Jin, G.; Zhao, J.; Han, J.; Zhu, S.; Zhang, J. Tetrahedron 2010, 66, 913. http://dx.doi.org/10.1016/j.tet.2009.11.092

11. Yadav, J. S.; Reddy, B. V. S.; Sadasiv, K.; Reddy, P. S. R. Tetrahedron Lett. 2002, 43, 3853. http://dx.doi.org/10.1016/S0040-4039(02)00681-0

12. El-Agrody, A. M.; Al-Ghamdi, A. M. Arkivoc 2011, (11), 134.

13. LianBin, Y.; Fan, X.; Qi, S. Chin. Sci. Bull. 2010, 55, 4108.

14. Reddy, B. V. S.; Medaboona, D.; Sridhar, B.; Singarapu, K. K. J. Org. Chem. 2013, 78, 8161. http://dx.doi.org/10.1021/jo4009657

15. Kumar, A.; Srivastava, S.; Gupta, G.; Chaturvedi, V.; Sinha, S.; Srivastava, R. ACS Comb. Sci. 2011, 13, 65. http://dx.doi.org/10.1021/co100022h

16. Chen, R.; Qian, C. Synth. Commun. 2002, 32, 2543. http://dx.doi.org/10.1081/SCC-120005937

17. Reddy, B. V. S.; Grewal, H. Tetrahedron Lett. 2011, 52, 761. http://dx.doi.org/10.1016/j.tetlet.2010.12.003

18. Khan, A. T.; Das, D. K.; Khan, M. M. Tetrahedron Lett. 2011, 52, 4539. http://dx.doi.org/10.1016/j.tetlet.2011.06.080

19. Kouznetsov, V. V. Tetrahedron 2009, 65, 2721. http://dx.doi.org/10.1016/j.tet.2008.12.059

20. Glushkov, V. A.; Tolstikov, A. G. Russ. Chem. Rev. 2008, 77, 137. http://dx.doi.org/10.1070/RC2008v077n02ABEH003749

21. Bello, D.; Ramon, R.; Lavilla, R. Curr. Org. Chem. 2010, 14, 332. http://dx.doi.org/10.2174/138527210790231883 
22. Povarov, L. S. Russ. Chem. Rev. 1967, 36, 656.

http://dx.doi.org/10.1070/RC1967v036n09ABEH001680

23. Fochi, M.; Caruana, L.; Bernardi, L. Synthesis 2014, 46, 135.

http://dx.doi.org/10.1055/s-0033-1338581

24. Jiang, X.; Wang, R. Chem. Rev. 2013, 113, 5515.

http://dx.doi.org/10.1021/cr300436a

25. Jiménez, O.; de la Rosa, G.; Lavilla, R. Angew. Chem. Int. Ed. 2005, 44, 6521. http://dx.doi.org/10.1002/anie.200501548

26. Narsaiah, A. V.; Reddy, A. R.; Reddy, B. V. S.; Yadav, J. S. Synth. Commun. 2010, 40, 1750 . http://dx.doi.org/10.1080/00397910903161736

27. Mahesh, M.; Reddy, C. V.; Reddy, K. S.; Raju, P. V. K.; Reddy, V. V. N. Synth. Commun. 2004, 34, 4089.

http://dx.doi.org/10.1081/SCC-200036586

28. Nagarapu, L.; Bantu, R.; Puligoundla, R. G. Eur. J. Chem. 2011, 2, 260. http://dx.doi.org/10.5155/eurjchem.2.2.260-265.263

29. Sabitha, G.; Reddy, M. S. K.; Arundhathi, K.; Yadav, J. S. ARKIVOC 2006, vi, 153.

30. Stridharan, V.; Avendaño, C.; Menéndez, J. C. Synthesis 2008, 1039.

31. Zhou, Z.; Xu, F.; Han, X.; Zhou, J.; Shen, Q.; Eur. J. Org. Chem. 2007, 31, 5265. http://dx.doi.org/10.1002/ejoc.200700288

32. Yadav, J. S.; Reddy, B. V. S.; Sunitha, V.; Reddy, K. S. Adv. Synth. Catal. 2003, 345, 1203. http://dx.doi.org/10.1002/adsc.200303101

33. Huang, Y.; Qiu, C.; Li, Z.; Feng, W.; Gan, H.; Liu, J.; Guo, K. ACS Sustainable Chem. Eng., 2016, 4, 47. http://dx.doi.org/10.1021/acssuschemeng.5b01379

34. Huang, D.; Xu, F.; Chen, T.; Wang, Y.; Lin, X. RSC Adv. 2013, 3, 573. http://dx.doi.org/10.1039/C2RA22796G

35. Liu, H.; Dagousset, G.; Masson, G.; Retailleau, P.; Zhu, J. J. Am. Chem. Soc. 2009, 131, 4598.

http://dx.doi.org/10.1021/ja900806q

36. Williams, D. B. G.; Cullen, A. J. Org. Chem. 2009, 74, 9509. http://dx.doi.org/10.1021/jo9020437

37. Simelane, S. B.; Kinfe, H. H.; Muller, A.; Williams, D. B. G. Org. Lett. 2014, 16, 4543. http://dx.doi.org/10.1021/o1502305j

38. Williams, D. B. G.; Simelane, S. B.; Lawton, M. Kinfe, H. H. Tetrahedron 2010, 66, 4573. http://dx.doi.org/10.1016/j.tet.2010.04.053

39. Williams, D. B. G.; Simelane, S. B.; Kinfe, H. H. Org. Biomol. Chem. 2012, 10, 5636. http://dx.doi.org/10.1039/c2ob25540e

40. Lin, W.-C.; Yang, D.-Y. Org. Lett. 2013, 15, 4862. http://dx.doi.org/10.1021/o1401932p 
41. Mandal, P.K.; Misra, A. K. Lett. Org. Chem. 2006, 3, 848.

http://dx.doi.org/10.2174/157017806779116978

42. Nagarajan, R; Chitra, S; Peramal, P. T. Tetrahedron. 2001, 57, 3419.

http://dx.doi.org/10.1016/S0040-4020(01)00719-0 